\title{
Projections of Polytopes and the Generalized Baues Conjecture*
}

\author{
J. Rambau and G. M. Ziegler \\ Fachbereich Mathematik, MA 6-1, Technische Universität Berlin, \\ Straße des 17. Juni 136, D-10623 Berlin, Germany \\ rambau/ziegler@math.tu-berlin.de
}

\begin{abstract}
Associated with every projection $\pi: P \rightarrow \pi(P)$ of a polytope $P$ is a partially ordered set of all "locally coherent strings": the families of proper faces of $P$ that project to valid subdivisions of $\pi(P)$, partially ordered by the natural inclusion relation. The "Generalized Baues Conjecture" posed by Billera et al. [4] asked whether this partially ordered set always has the homotopy type of a sphere of $\operatorname{dimension} \operatorname{dim}(P)-\operatorname{dim}(\pi(P))-1$. We show that this is true in the cases when $\operatorname{dim}(\pi(P))=1$ (see [4]) and when $\operatorname{dim}(P)-\operatorname{dim}(\pi(P)) \leq 2$, but fails in general. For an explicit counterexample we produce a nondegenerate projection of a five-dimensional, simplicial, 2-neighborly polytope $P$ with 10 vertices and 42 facets to a hexagon $\pi(P) \subseteq \mathbb{R}^{2}$. The construction of the counterexample is motivated by a geometric analysis of the relation between the fibers in an arbitrary projection of polytopes.
\end{abstract}

\section{Introduction}

In this paper we study the poset $\omega(P, \pi)$ of all "locally coherent strings" (defined below) associated with a projection of a convex polytope. In particular, we prove a new special case of the Generalized Baues Conjecture about the homotopy type of this poset, and disprove the Conjecture by explicit counterexamples in the general case.

The investigation of the posets $\omega(P, \pi)$ is motivated by problems that are concerned with the global (topological) structure of a restricted set of subdivisions of a fixed compact space. Such problems appear in very different frameworks, among them

- model theory of loop spaces (see [1] and [2]),

- spaces of triangulations of manifolds (see [15] for recent work),

- triangulations of point configurations and local transformations (see [10]-[12]),

* The first author was supported by the Deutsche Forschungsgemeinschaft (DFG), Grant We 1265/2-1. The second author was supported by a "Gerhard-Hess-Forschungsförderpreis" of the Deutsche Forschungsgemeinschaft (DFG). 
- extension spaces of oriented matroids (see [17]), and

- finite models of the finite-dimensional Grassmannians (see [13] and [14]).

The Generalized Baues Conjecture, whose precise setting we now introduce, directly applies to several of the situations we have just listed, and provides a prototypical model for the others.

Let $\pi: P \rightarrow \pi(P)$ be a projection of polytopes. Here we assume that $P$ is a $d$ polytope in $\mathbb{R}^{d}, \pi(P)$ is a $d^{\prime}$-polytope in $\mathbb{R}^{d^{\prime}}$, and $\pi: \mathbb{R}^{d} \rightarrow \mathbb{R}^{d^{\prime}}$ is an affine map. If $\pi$ maps more than one vertex of $P$ to a single point in $\pi(P)$ we call $\pi$ degenerate, while $\pi$ is weakly nondegenerate otherwise. If each affine dependence between projections of vertices $\pi\left(v_{1}\right), \pi\left(v_{2}\right), \ldots, \pi\left(v_{k}\right)$ is induced by an affine dependence between the vertices $v_{1}, \ldots, v_{k}$ in $P$, then we call $\pi$ (strongly) nondegenerate. The main objects of study in this paper are the following.

Definition 1.1. A locally $\pi$-coherent string-or a locally coherent string for short-is a collection $\mathcal{F}$ of nontrivial faces of $P$ (that is, faces different from $P$ and from $\emptyset$ ), such that:

- $\{\pi(F): F \in \mathcal{F}\}$ is a polytopal subdivision of $\pi(P)$ without repetitions, that is, the sets $\pi(F)$ are distinct polytopes which form a polytopal complex with union $\pi(P)$.

- $\pi(F) \subseteq \pi\left(F^{\prime}\right)$ implies $F=F^{\prime} \cap \pi^{-1}(\pi(F))$, for $F, F^{\prime} \in \mathcal{F}$.

The finite set of all locally $\pi$-coherent strings is partially ordered by

$$
\mathcal{F} \leq \mathcal{F}^{\prime} \quad: \Leftrightarrow \bigcup \mathcal{F} \subseteq \bigcup \mathcal{F}^{\prime}
$$

The resulting partially ordered set (poset) of locally $\pi$-coherent strings is denoted by $\omega(P, \pi)$. A string $\mathcal{F} \in \omega(P, \pi)$ is called

- tight if $\operatorname{dim}(\pi(F))=\operatorname{dim}(F)$ for all $F \in \mathcal{F}$, and

- globally $\pi$-coherent-or coherent for short-if a $\psi \in\left(\mathbb{R}^{d}\right)^{*} \backslash\{0\}$ exists such that $\pi$ can be factorized into

$$
\pi: P \stackrel{(\pi, \psi)}{\longrightarrow}\{(\pi(x), \psi(x)): \dot{x} \in P\} \stackrel{p r_{1}}{\longrightarrow} \pi(P),
$$

such that $(\pi, \psi)(\mathcal{F})$ is locally $p r_{1}$-coherent. The subposet of all coherent strings is denoted by $\omega_{\text {coh }}(P, \pi) \subseteq \omega(P, \pi)$.

For a part (a) of the following useful Lemma see Chapter 9 of [18]. Part (b) is an immediate consequence.

Lemma 1.2 (Properties of Tight Strings).

(a) A locally coherent string is minimal in $\omega(P, \pi(P))$ if and only if it is tight.

(b) If a tight locally coherent string is maximal, then it is an isolated element in $\omega(P, \pi(P))$.

Definition 1.1 is equivalent to the definition of the set of all $\pi$-induced subdivisions of $\pi(P)$, denoted " $S(P, \pi(P))$," in the paper of Billera et al. [4]. Since in general there 
may be many different locally $\pi$-coherent strings that determine the same polytopal subdivision of $\pi(P)$, we emphasize by our notation that one is dealing with objects in $P$ rather than with subdivisions of $\pi(P)$.

Billera and Sturmfels [5], [18, Theorem 9.6] showed that the subposet $\omega_{\text {coh }}(P, \pi)$ is isomorphic to the poset of proper faces of the fiber polytope $\Sigma(P, \pi)$ of the projection $\pi$, a convex polytope of dimension $d-d^{\prime}$. Thus the order complex (simplicial complex of chains, see [7]) of $\omega_{\mathrm{coh}}(P, \pi)$ is homeomorphic to a sphere of dimension $d-d^{\prime}-1$. In general, the poset $\omega(P, \pi)$ is strictly larger than $\omega_{\text {coh }}(P, \pi)$, and not homeomorphic to a sphere. (See, e.g., p. 297 of [18].) Huwever, in 1980 Baues conjectured in his work on a model theorem for loop spaces [2] (in somewhat different language) that for $d^{\prime}=1$ the poset $\omega(P, \pi)$ of all locally coherent strings is homotopy equivalent to the sphere $S^{d-2}$. In 1991 Billera $e$ al. extended this to the following conjecture.

Conjecture 1.3 (Generalized Baues Conjecture) [4], [16, Section 5]. For every projection $\pi: P \rightarrow \pi(P)$ of a d-polytope $P \subseteq \mathbb{R}^{d}$ to a $d^{\prime}$-polytope $\pi(P) \subseteq \mathbb{R}^{d^{\prime}}$, the poset $\omega(P, \pi)$ of all locally $\pi$-coherent strings is homotopy equivalent to the $\left(d-d^{\prime}-1\right)$ sphere.

Even stronger, $\omega_{\mathrm{coh}}(P, \pi)$ is a retract of $\omega(P, \pi)$ : the inclusion map

$$
\omega_{\mathrm{coh}}(P, \pi) \hookrightarrow \omega(P, \pi)
$$

is a homotopy equivalence.

Even for projections of reasonably small and simple polytopes, the poset of all locally coherent strings can be large and complicated. Up to now the main positive result, motivating the Generalized Baues Conjecture, was the following theorem, which settled the original conjecture by Baues [2].

Theorem 1.4 [4]. The Generalized Baues Conjecture holds for $d^{\prime} \leq 1$.

Actually, in [4] this is formulated only for the case where the projection is nondegenerate. However, the proof can be extended to the general case without greater difficulty.

Theorem 1.5 (Partial Results for Special Polytopes $P$ ).

- If $P$ is a simplex and $\operatorname{dim}(\pi(P)) \leq 2$, then the Generalized Baues Conjecture holds ([4], a proof was recently presented by Edelman and Reiner in [9]).

- If $P$ is a hypercube and $\operatorname{dim}(\pi(P)) \leq 2$ or $\operatorname{dim}(P)-\operatorname{dim}(\pi(P)) \leq 3$, then the Generalized Baues Conjecture holds (see [17]).

We refer to [5], [6], [14], and [16] for related discussions. Our main positive result is the following special case.

Theorem 1.6. The Generalized Baues Conjecture holds for $d-d^{\prime} \leq 2$.

After preliminary work on the structure of locally coherent strings (including a characterization theorem in terms of functions on the chamber complex) in Section 2, we prove Theorem 1.6 in Section 3. 
Theorem 1.7. The Generalized Baues Conjecture is false in general for $d^{\prime} \geq 2$ and $d-d^{\prime} \geq 3$.

In Section 4 we present a construction method for polytope projections that have isolated elements in their posets of all locally coherent strings, thus proving Theorem 1.7. In order to provide more geometric/combinatorial intuition for "what goes wrong here," we present explicit coordinates for two counterexamples in Section 5, together with simple, independent proofs that these polytope projections violate the Generalized Baues Conjecture. These proofs depend on "hands-on" knowledge of the face lattices of the polytopes, as can be obtained from Fourier-Motzkin elimination (or any similar convex hull algorithm).

The first example is one special instance of the construction method of Section 4. It is an extremely degenerate projection $\pi^{\text {deg: }} P^{\text {deg }} \rightarrow \pi\left(P^{\text {deg }}\right)=: Q^{\text {deg }}$, where $P^{\text {deg }}$ is a 5-polytope with 10 vertices and 36 facets and $Q^{\text {deg }}$ is a triangle. Each vertex of $P^{\text {deg }}$ is projected by $\pi^{\mathrm{deg}}$ either to a vertex or to the center of the triangle $Q^{\mathrm{deg}}$. In this case $\omega\left(P^{\text {deg }}, \pi^{\text {deg }}\right)$ has an isolated element.

The second example - obtained by perturbation of the vertices of the first-is a strongly nondegenerate projection $\pi: P \rightarrow \pi(P)=: Q$, where $P$ is a 2-neighborly, simplicial 5-polytope with 10 vertices and 42 (!) facets, and $Q$ is a hexagon. Here $\omega(P, \pi)$ is disconnected: the locally coherent strings of one connected component all have three special 2-faces of $P$ in common.

By Theorems 1.4 and 1.6 these counterexamples have both minimal dimension and codimension. They easily imply that the Generalized Baues Conjecture also fails in any higher dimension and codimension.

The most interesting cases of the Generalized Baues Conjecture that remain open are the following:

- $P$ is a simplex, and $\operatorname{dim}(\pi(P))>2$ (directly relevant for triangulations and their local transformations),

- $P$ is a hypercube, for $\operatorname{dim}(\pi(P))>2$ and $\operatorname{dim}(P)-\operatorname{dim}(\pi(P))>3$ (important for extension spaces of oriented matroids).

\section{Functions on the Chamber Complex}

In this section we point out two crucial facts. The first one describes a basic property of the chamber complex of a polytope projection, the second one is a "local coherence condition" in terms of the normal fans of the fibers of the projection.

Given any linear or affine function $\psi$ on a space that contains the polytope $P$, we use $P^{\psi}$ to denote the set of all points in $P$ on which $\psi$ is maximal. This set $P^{\psi}$ is a face of $P$, and all nonempty faces of $P$ have this form ( $\psi=0$ corresponds to $P$ itself). We use $L(P)$ to denote the face lattice of $P$ : the set of all faces of $P \subseteq \mathbb{R}^{d}$, partially ordered by inclusion. This includes the trivial faces $\emptyset$ and $P$.

For a polytope projection $\pi: P \rightarrow \pi(P)=: Q$ as above, the chamber complex $\Gamma$ is the set of intersections of all images of faces of $P$ that contain a given point in $Q$, that is,

$$
\Gamma:=\{\sigma(q): q \in Q\}
$$


where

$$
\sigma(q):=\bigcap\{\pi(F): q \in \pi(F), F \in L(P)\}
$$

is the chamber of $q \in Q$. (It can be shown that $\Gamma$ is a polytopal complex subdividing $Q$. The chamber complex $\Gamma$ is the common refinement of all $\pi$-coherent subdivisions of $Q$, and therefore shellable.)

There is no loss of generality if we assume from now on that the projection map $\pi: \mathbb{R}^{d} \rightarrow \mathbb{R}^{d^{\prime}}$ is the restriction to the last $d^{\prime}$ coordinates. For any $q \in Q$ the fiber of $q$ is the polytope

$$
P_{q}:=\left\{x \in \mathbb{R}^{d-d^{\prime}}:(x, q) \in P\right\} .
$$

Thus we consider the fibers as full-dimensional polytopes $P_{q}$ in the (fixed) vector space $\mathbb{R}^{d-d^{\prime}}$. Whenever we need to interpret a fiber as a subset of $\mathbb{R}^{d}$ we write $i_{q}\left(P_{q}\right)$, where $i_{q}(x):=(x, q) \in \mathbb{R}^{d}$. The (surjective) map $i_{q}^{*}:\left(\mathbb{R}^{d}\right)^{*} \rightarrow\left(\mathbb{R}^{d-d^{\prime}}\right)^{*}$ is as usual defined by $i_{q}^{*}(\alpha)(x):=\alpha\left(i_{q}(x)\right)=\alpha(x, q)$.

The nonempty faces of the fibers $P_{q}$ can be represented in the form $P_{q}^{\psi}$, where $\psi$ is a linear functional $\psi \in\left(\mathbb{R}^{d-d^{\prime}}\right)^{*}$. Now if $P_{q}^{\psi}$ is any nonempty face of a fiber $P_{q}$, then we use $[\psi]$ to denote the (closed, polyhedral) cone in $\left(\mathbb{R}^{d-d^{\prime}}\right)^{*}$ of all linear functions that are maximal on the face $P_{q}^{\psi}$ of $P_{q}$. This set $[\psi]$ is the normal cone of the face $P_{q}^{\psi}$. If $q^{\prime}$ is another point that lies in the relative interior of the same chamber of the chamber complex as $p$, then the normal cones of the face $P_{q}^{\psi}$ of $P_{q}$ and $P_{q^{\prime}}^{\psi}$ of $P_{q^{\prime}}$ coincide (that is, the fibers $P_{q}$ and $P_{q^{\prime}}$ are normally equivalent, see, e.g., [5]). Thus we can use the notation $[\psi]_{\sigma}$ for the normal cone of the face that $\psi$ defines in the fiber, called the normal cone over $\sigma$ induced by $\psi$. Moreover, let $N(\sigma)$ denote the fan consisting of all normal cones over $\sigma$, the normal fan over $\sigma$ (that is, the normal fan of the fiber over a point in the relative interior of $\sigma$ ).

For each face $P_{q}^{\psi}$ of a fiber $P_{q}$ there is a unique minimal face of $P$ that contains $P_{q}^{\psi}$ (the intersection of all faces that contain $P_{q}^{\psi}$ ). We use $F_{q, \psi}$ to denote this face of $P$ corresponding to the face $P_{q}^{\psi}$ of $P_{q}$. For its normal cone in $\left(\mathbb{R}^{d}\right)^{*}$ we use the notation $C_{P}\left(F_{q, \psi}\right)$. Figure 1 depicts the situation for $d=2$ and $d^{\prime}=1$.

The following lemma collects the elementary basic facts.

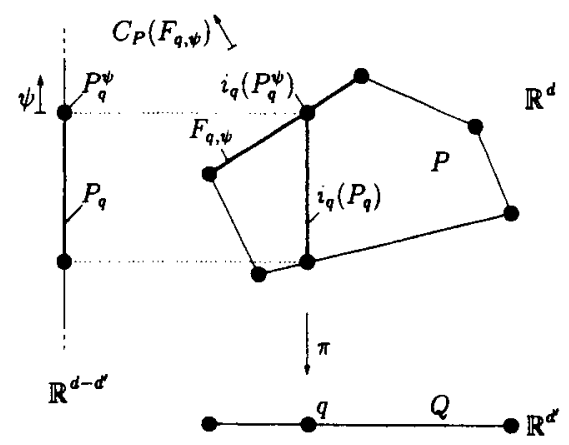

Fig. 1. The face $F_{q, \psi}$ induced by $\psi \in\left(\mathbb{R}^{d-d^{\prime}}\right)^{*}$. 
Lemma 2.1 (Basic Facts). The faces of the polytope $P$, of the fibers $P_{q}$, and the chambers $\sigma \in \Gamma$, are related as follows:

(i) The chamber of $q \in Q$ is given by

$$
\sigma(q)=\bigcap_{\psi \in\left(\mathbb{R}^{d-d^{\prime}}\right)^{*}} \pi\left(F_{q, \psi}\right)
$$

(ii) For all $q \in Q$ and $\psi, \psi^{\prime} \in\left(\mathbb{R}^{d-d^{\prime}}\right)^{*}$,

$$
P_{q}^{\psi}<P_{q}^{\psi^{\prime}} \Leftrightarrow F_{q, \psi}<F_{q, \psi^{\prime}} .
$$

(iii) For all $q, q^{\prime} \in Q$ and $\psi \in\left(\mathbb{R}^{d-d^{\prime}}\right)^{*}$,

$$
\begin{aligned}
q^{\prime} \in \text { relint } \pi\left(F_{q, \psi}\right) & \Rightarrow F_{q^{\prime}, \psi}=F_{q, \psi}, \\
q^{\prime} \in \partial \pi\left(F_{q, \psi}\right) & \Rightarrow F_{q^{\prime}, \psi} \subset F_{q, \psi}, \\
\pi\left(F_{q^{\prime}, \psi}\right) \subset \pi\left(F_{q, \psi}\right) & \Leftrightarrow \quad F_{q^{\prime}, \psi} \subset F_{q, \psi}, \\
\pi\left(F_{q_{1}, \psi} \cap \cdots \cap F_{q_{k}, \psi}\right) & =\pi\left(F_{q_{1}, \psi}\right) \cap \cdots \cap \pi\left(F_{q_{k}, \psi}\right) .
\end{aligned}
$$

(iv) Let $q^{\prime} \in \sigma(q), q \in Q, x \in P_{q}^{\psi}, x^{\prime} \in P_{q^{\prime}}^{\psi}$, and $\alpha \in C_{P}\left(F_{q, \psi}\right)$. Then

$$
i_{q^{\prime}}^{*}(\alpha)\left(x^{\prime}\right)=i_{q}^{*}(\alpha)(x) .
$$

(v) The normal cone $C_{P}\left(F_{q, \psi}\right)$ of $F_{q, \psi}$ in $\left(\mathbb{R}^{d}\right)^{*}$ is mapped by $i_{q}^{*}$ onto the normal cone of $P_{q}^{\psi}$ in $\left(\mathbb{R}^{d-d^{\prime}}\right)^{*}$.

(vi) For each face $F$ of $P$ there are $q \in Q$ and $\psi \in\left(\mathbb{R}^{d-d^{\prime}}\right)^{*}$ such that

$$
F=F_{q, \psi}
$$

As a corollary of (iii) we get that if $\psi$ is fixed, then the face $F_{q . \psi}$ does not change if $q$ moves in the relative interior of the chamber $\sigma(q)$. Hence with each chamber $\sigma$ and each functional $\psi$ we can associate a well-defined face of $P$, via

$$
F_{\sigma, \psi}:=F_{q, \psi}, \quad q \in \operatorname{relint}(\sigma) .
$$

The following "normal fan relation" of the chamber was used in the special case $d^{\prime}=1$ by Billera et al. [4] in their proof of the Baues Conjecture. Here we state its general validity.

Lemma 2.2. If $\tau$ is a face of $\sigma$ in the chamber complex $\Gamma$, then for each $\psi \in\left(\mathbb{R}^{d-d^{\prime}}\right)^{*}$ the normal cone over $\tau$ defined by $\psi$ is contained in the corresponding normal cone over $\sigma$ :

$$
\tau<\sigma \in \Gamma \Rightarrow[\psi]_{\sigma} \subseteq[\psi]_{\tau}
$$

Hence, the normal fan over $\sigma$ is a refinement of the normal fan over $\tau$ :

$$
N(\sigma) \preceq N(\tau) .
$$




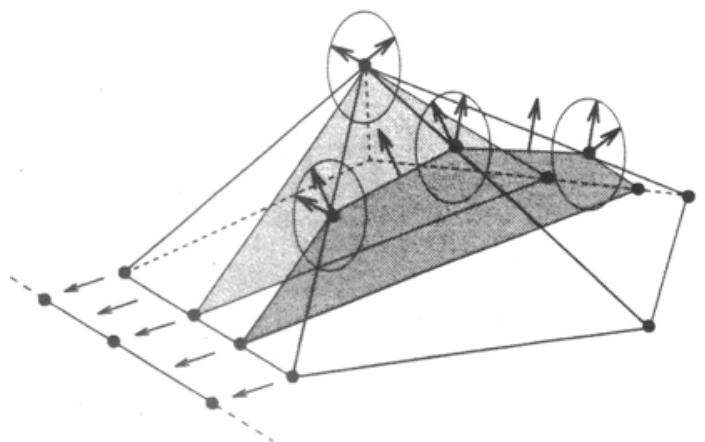

(a)

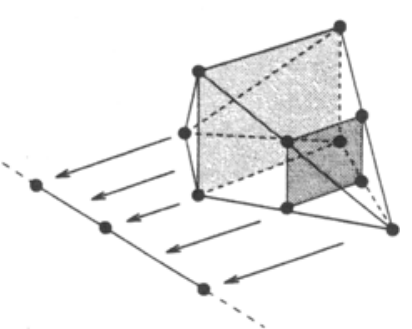

(b)

Fig. 2. The normal fan relation.

Proof. Let $\varphi=i_{q}^{*}(\alpha)$ be a linear functional on $\left(\mathbb{R}^{d-d^{\prime}}\right)^{*}$ in $[\psi]_{\sigma}$ with $q \in \operatorname{relint}(\sigma)$ and some $\alpha$ in the normal cone $C_{P}\left(F_{\sigma, \psi}\right)$ of $F_{\sigma, \psi}$ in $P$ by Lemma 2.1(v). Then

$$
\varphi=i_{q}^{*}(\alpha) \in i_{q}^{*}\left(C_{P}\left(F_{\sigma, \psi}\right)\right)
$$

However, this is contained in $i_{q^{\prime}}^{*}\left(C_{P}\left(F_{\sigma, \psi}\right)\right)$ for each $q^{\prime} \in \sigma$ (Lemma 2.1(iv)), especially for $q^{\prime} \in \tau$.

We know from Lemma 2.1(iii) that if $\tau$ is a face of $\sigma$, then $F_{\tau, \psi}$ is a face of $F_{\sigma, \psi}$ for all $\psi \in\left(\mathbb{R}^{d-d^{\prime}}\right)^{*}$. Hence, again from Lemma $2.1(\mathrm{v})$ we derive

$$
i_{q^{\prime}}^{*}\left(C_{P}\left(F_{\sigma, \psi}\right)\right) \subseteq i_{q^{\prime}}^{*}\left(C_{P}\left(F_{\tau, \psi}\right)\right)=[\psi]_{\tau},
$$

which completes the proof.

Remark 2.3. In general we cannot expect a strict refinement (see Fig. 2), because the map $i_{q^{\prime}}^{*}$ does not preserve strict inclusions if the projection is degenerate. However, if we restrict ourselves to nondegenerate projections, then the cone inclusion has to be proper for at least one $\psi \in\left(\mathbb{R}^{d-d^{\prime}}\right)^{*}$, and therefore the fan refinement is strict.

The following proposition describes the relations between the fibers over adjacent chambers even metrically.

Proposition 2.4. Let $\sigma \in \Gamma$ be a chamber with vertices $v_{1}, \ldots, v_{k}$ and

$$
q=\sum_{i=1}^{k} \lambda_{i} v_{i}
$$

with $\lambda_{i} \geq 0$ and $\sum_{i=1}^{k} \lambda_{i}=1$. Then $P_{q}$ is the Minkowski sum of the fibers over the vertices of $\sigma$, scaled as in the representation of $q$ in $\sigma$,

$$
P_{q}=\sum_{i=1}^{k} \lambda_{i} P_{v_{i}} .
$$


Proof. Consider the polytope projection

$$
\pi_{\sigma}: P_{\sigma}:=\pi^{-1}(\sigma) \rightarrow \sigma .
$$

In this very special case the fiber over each vertex $v_{i}$ of $\sigma$ is the convex hull of vertices $v_{i, 1}, \ldots, v_{i, l(i)}$ of $P_{\sigma}$ and these are the only vertices of $P_{\sigma}$. This yields the claim after a straightforward computation.

Corollary 2.5. The normal fan over the relative interior of a chamber $\sigma \in \Gamma$ is exactly the common refinement of the normal fans over the faces of $\sigma$.

Any locally coherent string can be interpreted as a function which associates a face of $P_{q}$ to every point $q \in Q$ in some "locally coherent" way. This selection must be constant (in the sense that the same face $F_{\sigma, \psi}$ is chosen) in the relative interior of every chamber. No locally coherent string can contain a whole $d^{\prime}$-dimensional fiber $P_{q}$ for some $q \in Q$, because this would imply that $P$ itself is contained in that string. Complete fibers $P_{q}$ of dimension smaller than $d^{\prime}-$ e.g., for $q$ in the boundary of $Q$-can always be expressed by nonzero normal vectors. (For example, if a fiber consists only of one vertex any nonzero vector will do the job.) Hence, we interpret the selection functions as functions from $\Gamma$ to $S^{d-d^{\prime}-1}$, where $\psi_{\sigma} \in S^{d-d^{\prime}-1}$ induces a face of a fiber over $\sigma$-which is a proper one whenever the fiber is full-dimensional-and therefore a proper face of $P$.

The following criterion (see [4] for the case $d^{\prime}=1$ ) describes the admissible selection functions in terms of normal cones.

Proposition 2.6 (Cone Condition). A function

$$
\psi:\left\{\begin{array}{l}
\Gamma \rightarrow S^{d-d^{\prime}-1}, \\
\sigma \mapsto \psi_{\sigma}
\end{array}\right.
$$

defines a locally $\pi$-coherent string of $Q$ via

$$
\mathcal{F}(\psi):=\left\{F_{\sigma, \psi_{\sigma}}: \sigma \in \Gamma\right\}
$$

if and only iffor all $\sigma, \tau \in \Gamma$ with $\tau<\sigma$ one has

$$
\operatorname{relint}\left[\psi_{\sigma}\right]_{\sigma} \subseteq \operatorname{relint}\left[\psi_{\tau}\right]_{\tau} .
$$

Furthermore, every locally coherent string arises from a selection function $\psi$ in this way. Two functions $\psi$ and $\psi^{\prime}$ define the same string, $\mathcal{F}(\psi)=\mathcal{F}\left(\psi^{\prime}\right)$, if and only if $\left[\psi_{\sigma}\right]_{\sigma}=\left[\psi_{\sigma}^{\prime}\right]_{\sigma}$ holds for all $\sigma \in \Gamma$.

The proof is a careful check of definitions, where Lemma 2.1 yields the necessary details.

Definition 2.7. A function $\psi$ as in Proposition 2.6 is called locally coherent. Two functions $\psi, \psi^{\prime}$ are equivalent if they define the same locally coherent string. In this case we write

$$
[\psi]=\left[\psi^{\prime}\right]
$$

for their equivalence class. 
Because of Lemma 2.2 the crucial function values are just those over the chambers of maximal dimension.

Proposition 2.8 (Pairwise Cone Condition). The cone condition in Proposition 2.6 is equivalent to the following:

$$
\left[\psi_{\sigma_{1}}\right]_{\sigma_{1} \cap \sigma_{2}}=\left[\psi_{\sigma_{2}}\right]_{\sigma_{1} \cap \sigma_{2}}
$$

for all $d^{\prime}$-dimensional chambers $\sigma_{1}, \sigma_{2} \in \Gamma$ such that $\sigma_{1} \cap \sigma_{2} \neq \emptyset$. Any function that respects the pairwise cone condition for the chambers of dimension $d^{\prime}$ can be completed to a locally coherent function.

Figure 3 illustrates Propositions 2.6 and 2.8 for the situation $d=3$ and $d^{\prime}=1$.

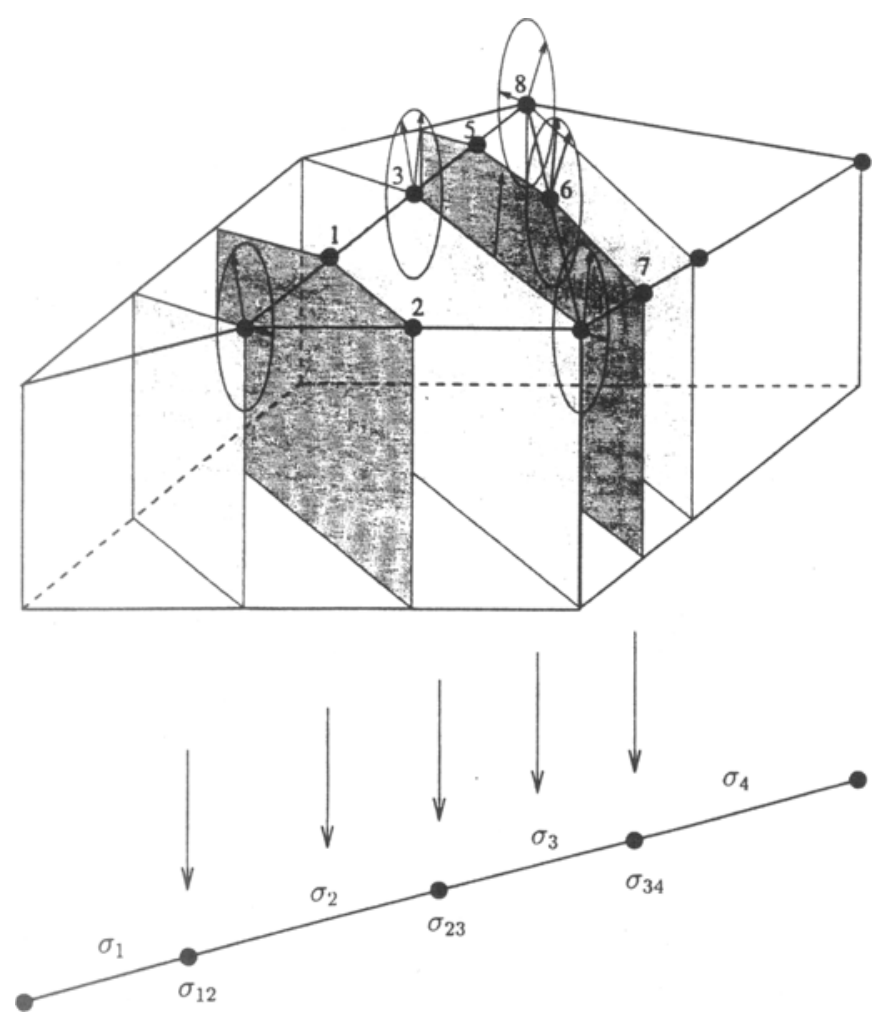

Fig. 3. The pairwise cone condition. For example, a choice of 1 over $\sigma_{2}$ and 5 over $\sigma_{3}$ is locally coherent and would imply the choice of 3 over $\sigma_{23}$. If 2 is chosen over $\sigma_{2}$, then 5 is not a consistent choice over $\sigma_{3}$. However, in this case 6 or 7 are "good choices" over $\sigma_{3}$-with respect to the pairwise cone condition-which both determine 4 over $\sigma_{23}$. Observe that, for example, the open normal cone at 6 is the intersection of the open normal cones at 8 and 4 (compare Corollary 2.5 ). 


\section{Validity in Low Codimension}

In this section we prove Theorem 1.6, by presenting an explicit retraction of the following models of the order complexes of $\omega(P, \pi)$ and $\omega_{\text {coh }}(P, \pi)$, namely

$$
\Omega=\left\{\psi \in\left(S^{1}\right)^{\Gamma}:\left[\psi_{\sigma}\right]_{\tau}=\left[\psi_{\tau}\right]_{\tau} \text { for all } \tau \leq \sigma \in \Gamma\right\}
$$

and

$$
\Omega_{\text {coh }}=\left\{\psi \in\left(S^{1}\right)^{\Gamma}: \psi_{\sigma}=\psi_{\tau} \text { for all } \tau, \sigma \in \Gamma\right\} .
$$

The topology of the order complexes coincides with the topology induced by the canonical metric on $\Omega, \Omega_{\text {coh }} \subseteq\left(S^{1}\right)^{\Gamma}$, induced from $\left(S^{1}\right)^{\Gamma}$ viewed as a product of copies of the metric space $S^{1}$.

Let $\sigma \in \Gamma$. From now on we call two values $\psi_{1}$ and $\psi_{2}$ in $S^{1}$ locally coherent with respect to $\sigma$, if

$$
\left[\psi_{1}\right]_{\sigma}=\left[\psi_{2}\right]_{\sigma}
$$

A function

$$
\psi: \Gamma \rightarrow S^{1}
$$

defines a locally coherent string if and only if all function values of intersecting chambers are pairwise locally coherent with respect to the intersection of their preimages. (This is the pairwise cone condition of Proposition 2.8.)

The crucial observation for the situation in codimension 2 is that the local coherence property reduces to a distance property for function values in the universal cover of $S^{1}$ : if we replace two locally coherent function values by values in the closed interval they span, then they stay locally coherent. In higher codimensions this fails in general.

Proof of Theorem 1.6 (in seven steps).

Step 1 . From now on we write $q_{\sigma}$ for the barycenter of the chamber $\sigma \in \Gamma$. For a function

$$
\psi:\left\{\begin{array}{l}
\Gamma \rightarrow S^{1}, \\
\sigma \mapsto \psi_{\sigma}
\end{array}\right.
$$

that defines a locally coherent string let

$$
\hat{\psi}: Q \rightarrow S^{1}
$$

be the unique piecewise linear function on $Q$ with

$$
\hat{\psi}\left(q_{\sigma}\right)=\psi_{\sigma}
$$

for all chambers $\sigma \in \Gamma$. Here "piecewise linear" means that whenever $q$ is in the simplex spanned by the barycenters of the chambers $\sigma_{i}, i=1, \ldots, k$, with barycentric coordinates $\lambda_{1}, \ldots, \lambda_{k} \geq 0$ and $\sum_{i=1}^{k} \lambda_{i}=1$, its function value is given by

$$
\hat{\psi}(q)=\frac{\sum_{i=1}^{k} \lambda_{i} \hat{\psi}\left(q_{\sigma_{i}}\right)}{\left\|\sum_{i=1}^{k} \lambda_{i} \hat{\psi}\left(q_{\sigma_{i}}\right)\right\|} .
$$


This yields a well-defined continuous function: the function $\psi$ defines a locally coherent string and thus the function values on pairwise adjacent chambers lie inside some open hemisphere in $S^{1}$ (see Corollary 2.5).

Step 2. For the rest of the proof let $\sigma_{0}$ be a fixed chamber of $\Gamma$, let $\psi: \Gamma \rightarrow S^{1}$ be a locally coherent function, and let $\psi_{0}:=\psi_{\sigma_{0}}$ be its value for $\sigma_{0}$. For $\lambda \in S^{1}$ let

$$
\Phi_{\lambda}:\left\{\begin{aligned}
\{z \in \mathbb{C}:\|z\|=1\} & \rightarrow S^{1}, \\
1 & \mapsto \lambda
\end{aligned}\right.
$$

be an isometry that coordinatizes $S^{1}$. Let

$$
w:[0,1] \rightarrow Q
$$

be a path in $Q$ that starts at $q_{\sigma_{0}}$. Then

$$
\hat{\psi}_{*}(w):\left\{\begin{aligned}
{[0,1] } & \rightarrow S^{1} \\
t & \mapsto(\hat{\psi} \circ w)(t)
\end{aligned}\right.
$$

is a path in $S^{1}$ that starts at $\psi_{0}$.

Step 3. Let

$$
p:\left\{\begin{aligned}
\mathbb{R} & \rightarrow\{z \in \mathbb{C}:\|z\|=1\}, \\
t & \mapsto \exp (2 \pi i t)
\end{aligned}\right.
$$

be the universal covering of $\{z \in \mathbb{C}:\|z\|=1\}$ and let

$$
p_{\lambda}:\left\{\begin{aligned}
\mathbb{R} & \rightarrow S^{1}, \\
t & \mapsto\left(\Phi_{\lambda} \circ p\right)(t)
\end{aligned}\right.
$$

be the universal covering of $S^{1}$ where the parameter $\lambda$ describes different coordinate systems on $S^{1}$. For a path

$$
u:[0,1] \rightarrow S^{1}
$$

with $u(0)=\lambda$ let

$$
L_{\lambda}(u):=L_{p_{\lambda}}(u, 0):[0,1] \rightarrow \mathbb{R}
$$

be its lifting with $L_{\lambda}(u)(0)=0$. We know from the theory of coverings that liftings of paths that are homotopic relative to $\partial[0,1]$ have the same endpoint.

Step 4. We now lift the "distance" between the considered function values to $\mathbb{R}$ in order to get maximum and minimum values.

Definition 3.1. We define the twist of $\psi$ to be the following function:

$$
\text { twist }_{\psi}:\left\{\begin{array}{l}
\Gamma \rightarrow \mathbb{R}, \\
\sigma \mapsto L_{\psi_{0}}\left(\hat{\psi}_{*}\right)(w)(1),
\end{array}\right.
$$

where $w:[0,1] \rightarrow Q$ is a path from $q_{\sigma_{0}}$ to $q_{\sigma}$. 


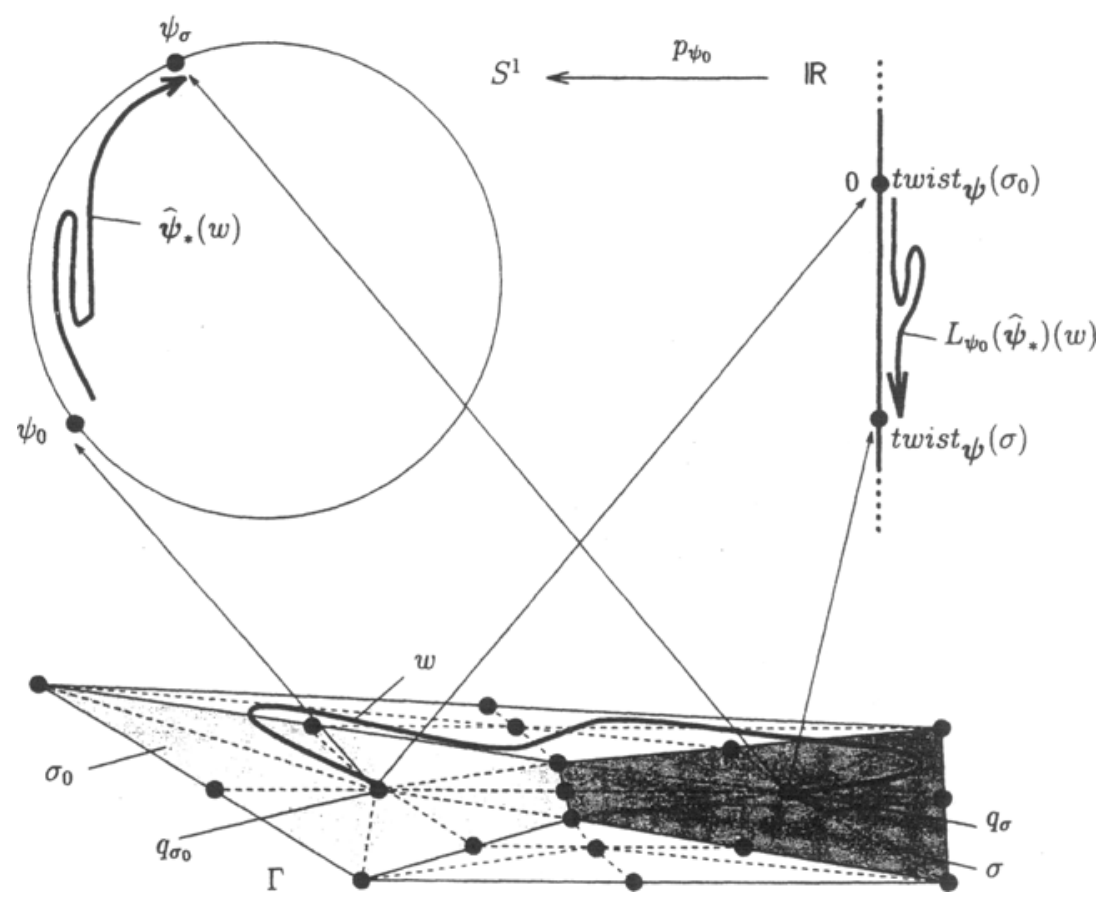

Fig. 4. The twist of $\sigma$.

In other words: coordinatize $S^{1}$ properly, take a path from the barycenter of $\sigma_{0}$ to the barycenter of $\sigma$, consider the corresponding path induced by the piecewise linear extension $\hat{\psi}$ of $\psi$, and take the endpoint of its lifting to $\mathbb{R}$. This is well defined by step 3 because all paths in $Q$ are homotopic. From the definition we get that $t w i s t_{\psi}\left(\sigma_{0}\right)=0$. Figure 4 shows the twist of the chamber $\sigma$.

A locally coherent string is globally coherent if and only if it can be described by a function $\psi$ with wist $_{\psi}(\Gamma)=\{0\}$. In addition we have $p_{\psi_{0}} \circ$ twist $_{\psi}=\hat{\psi}$, which makes it possible to recover the function $\psi$ from its twist or to define a new function $\psi^{\prime}$ by simply changing the twist of $\psi$ (with twist of $\sigma_{0}$ unchanged) and projecting it via $p_{\psi_{0}}$.

Step 5. The following lemma shows that local coherence in this special case is preserved under "pushing together" lifted function values-this is the crucial point that cannot be generalized to higher codimension.

Lemma 3.2. Let $\psi: \Gamma \rightarrow S^{1}$ be a locally coherent function, in particular $\psi_{\sigma_{1}}, \psi_{\sigma_{12}}$, and $\psi_{\sigma_{2}}$ are painwise locally coherent with respect to $\sigma_{12}:=\sigma_{1} \cap \sigma_{2}$. Without loss of generality, let twist $\psi_{\psi}\left(\sigma_{1}\right)<$ twist $_{\psi}\left(\sigma_{2}\right)$. Then each pair of values $\psi_{1}, \psi_{2}$ contained in the arc

$$
p_{\psi_{0}}\left(\left[\text { twist }_{\psi}\left(\sigma_{1}\right), \text { twist }_{\psi}\left(\sigma_{2}\right)\right]\right) \subset S^{1}
$$

is locally coherent with respect to $\sigma_{12}$ as well. 
Proof. Let $w_{0,1}:[0,1] \rightarrow Q$ be an arbitrary path from $q_{\sigma_{0}}$ to $q_{\sigma_{1}}$, and let $w_{1,2}:[0,1] \rightarrow$ $Q$ be the polygonal path which leads straight from $q_{\sigma_{1}}$ to $q_{\sigma_{12}}$ and then straight from $q_{\sigma_{12}}$ to $q_{\sigma_{2}}$. (The following will not depend on parametrization.) Because of local coherence over $\sigma_{12}$ we have

$$
\begin{aligned}
& \psi_{\sigma_{1}} \in \operatorname{relint}\left[\psi_{\sigma_{12}}\right]_{\sigma_{12}}, \\
& \psi_{\sigma_{2}} \in \operatorname{relint}\left[\psi_{\sigma_{12}}\right]_{\sigma_{12}}, \\
& \psi_{\sigma_{12}} \in \operatorname{relint}\left[\psi_{\sigma_{12}}\right]_{\sigma_{12}}
\end{aligned}
$$

By definition, each $q$ on the straight line from $q_{\sigma_{1}}$ to $q_{\sigma_{12}}$ is mapped by $\hat{\psi}$ to a point between $\hat{\psi}\left(q_{\sigma_{1}}\right)=\psi_{\sigma_{1}}$ and $\hat{\psi}\left(q_{\sigma_{12}}\right)=\psi_{\sigma_{12}}$. ("Between" is well defined because all these points lie in the same pointed cone $\left[\psi_{\sigma_{12}}\right]_{\sigma_{12}}$.) Analogously, each $q$ on the straight line from $q_{\sigma_{12}}$ to $q_{\sigma_{2}}$ is mapped by $\hat{\psi}$ to a point between $\hat{\psi}\left(q_{\sigma_{12}}\right)=\psi_{\sigma_{12}}$ and $\hat{\psi}\left(q_{\sigma_{2}}\right)=\psi_{\sigma_{2}}$.

Hence we get

$$
\hat{\psi}_{*}\left(w_{1,2}\right)(t) \in \operatorname{relint}\left[\psi_{\sigma_{12}}\right]_{\sigma_{12}} \quad \text { for all } t \in[0,1] .
$$

Therefore, if we compute the twist of $\psi_{1}$ and $\psi_{2}$ using the paths $w_{0,1}$ and $w_{0,1} \cdot w_{1,2}$ (the concatenation of $w_{0,1}$ and $w_{1,2}$ ), we get that all values in the interval

$$
\left[\text { twist }_{\psi}\left(\sigma_{1}\right), \text { twist }_{\psi}\left(\sigma_{2}\right)\right]
$$

project into the open cone relint $\left[\psi_{\sigma_{12}}\right]_{\sigma_{12}}$, and hence produce local coherent pairs.

If a twist is extremal, then there is only one direction in $\mathbb{R}$ with other twist values. That means we can "retwist" all chambers that yield this extremal value until their twist meets the next occurring different twist. So at the next step we introduce a "twist cutoff" homotopy.

Step 6. Let $M(\psi)$ be the maximum of all absolute values of $\psi$-twists taken over all chambers $\sigma \in \Gamma$. Define

$$
\text { twist }_{\psi}(\sigma, t):\left\{\begin{aligned}
\Gamma \times[0,1] & \rightarrow \mathbb{R} \\
(\sigma, t) & \mapsto \max \left\{\min \left\{\text { twist }_{\psi}(\sigma), t M(\psi)\right\},-t M(\psi)\right\}
\end{aligned}\right.
$$

Step 7. Now we are in position to define the final "retwist" homotopy. Let

$$
\psi_{t}:\left\{\begin{aligned}
\Gamma \times[0,1] & \rightarrow S^{1}, \\
(\sigma, t) & \mapsto p_{\psi_{0}}\left(t_{w i s t_{\psi}}(\sigma, t)\right) .
\end{aligned}\right.
$$

Then $\psi_{1}(\sigma)=\psi_{\sigma}$ and $\psi_{0}(\sigma)=\psi_{0}$ for all $\sigma \in \Gamma$. Hence, $\psi_{1}=\psi \in \Omega$ and $\psi_{0} \in \Omega_{\mathrm{coh}}$. This yields the desired retraction

$$
H:\left\{\begin{aligned}
\Omega \times[0,1] & \rightarrow S^{1}, \\
(\psi, t) & \mapsto \psi_{t}
\end{aligned}\right.
$$

with

$$
H(\Omega, 1)=\mathrm{id}_{\Omega} \text { and } H(\Omega, 0)=\Omega_{\text {coh }} .
$$

This retraction is continuous in $t$ by definition. It is continuous in $\psi$ because it contracts distances between functions according to the maximum metric. 
This proof and the proof of Theorem 1.4 by Billera et al. [4] suggest a duality between the geometric situations in the $\operatorname{case} \operatorname{dim}(Q)=1$ and the case $\operatorname{dim}(P)-\operatorname{dim}(Q)=2$, as would be expected from an oriented matroid perspective (see also Billera et al. [3]):

- In the case of dimension 1 the polytope $Q$ is linearly ordered and therefore has a "maximum chamber" with a local coherence condition only in one direction. The retraction can start at this chamber moving its function value to that of the next adjacent chamber, no matter what the dimension of the image sphere is.

- In the case of codimension 2 the chambers can yield a very complicated structure of local coherence conditions between their function values, but in this case the lifting of the image of this structure can be easily retracted in $\mathbb{R}$ starting from its boundary, i.e., from the extremal values.

Analysis of the key points in the proof of Theorem 1.6 also led us to the crucial structures for the counterexamples in Sections 4 and 5.

\section{How To Construct a Counterexample}

In this section we introduce the main idea for the construction of a counterexample in $\operatorname{dimension} \operatorname{dim}(Q)=2$ and codimension $\operatorname{dim}(P)-\operatorname{dim}(Q)=3$. We start with a configuration of three two-dimensional chambers $\sigma_{1}, \sigma_{2}, \sigma_{3}$ that form a subdivision $\Gamma$ of $Q$ (see Fig. 5(a)). The corresponding edges in the boundary of $Q$ are $\tau_{1}, \tau_{2}$, and $\tau_{3}$. We denote $\sigma_{i} \cap \sigma_{j}$ by $\sigma_{i j}$, and thus the inner vertex $\sigma_{1} \cap \sigma_{2} \cap \sigma_{3}$ by $\sigma_{123}$. Analogously, we set $\tau_{i} \cap \tau_{j}=: \tau_{i j}$.

We want to construct functions $\psi: \Gamma \rightarrow S^{2}$ that satisfy the "local coherence condition" (Proposition 2.6) with respect to the fiber structure of some polytope projection. First we assign to each $\sigma_{i}$ a fixed value $\psi_{\sigma_{i}}$ in $S^{2}$ such that the cone spanned by the $\psi_{\sigma_{i}}$ in $\left(\mathbb{R}^{3}\right)^{*}$ is full-dimensional. Since $\psi$ ought to be locally coherent this leads to several restrictions on the possible structures of the normal fans over the chambers.

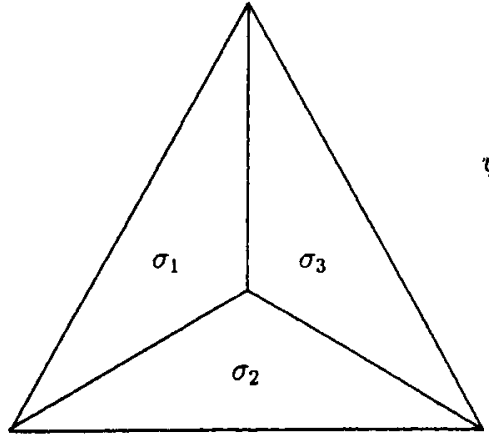

(a)

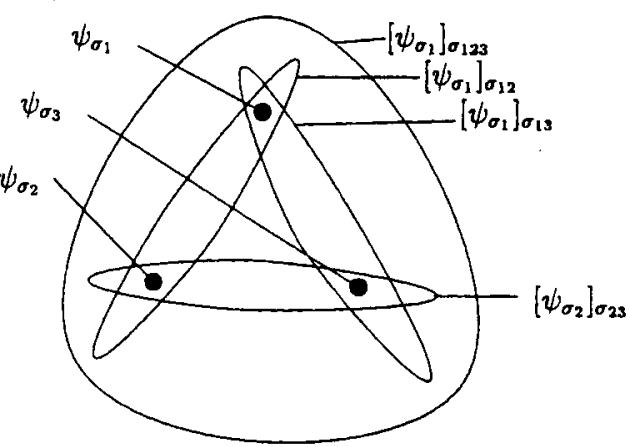

(b)

Fig. 5. A simple chamber complex (a) and a sketch for a possible "locally coherent" choice of function values on this complex (b). 
There is a consistent choice for $\psi_{\sigma_{12}}$ only if $\psi_{\sigma_{1}}$ and $\psi_{\sigma_{2}}$ lie in the same open cone of the normal fan over relint $\left(\sigma_{12}\right)$. In general, this open cone (which describes the correct selections for $\psi_{\sigma_{12}}$ ) does not contain $\psi_{\sigma_{3}}$. These cones are the crucial ones because, for local coherence at the inner vertex $\sigma_{123}$, we just have to choose a vector in the open cone of the normal fan over $\sigma_{123}$ that contains the rest of the configuration, which is always possible (see Lemma 2.2). The generic topological picture of the situation in the sphere $S^{2}$ is as in Fig. 5(b), which is a superposition of cones from the normal fans over $\sigma_{i j}$ and $\sigma_{123}$.

If the vectors $\psi_{\sigma_{i}}$ are in general position with respect to some fiber structure, then the locally $\pi$-coherent string $\mathcal{F}_{0}$ they determine in a polytope projection that induces this fiber structure is tight. In the following we describe what "has to go wrong" to get a fiber structure in which this tight string is not dominated by a nontight one $\mathcal{F}>\mathcal{F}_{0}$. (In this case the tight string is stuck: this is the situation of Lemma 1.2(b).)

To get from $\mathcal{F}_{0}$ to $\mathcal{F}$, we have to move at least one of the vectors $\psi_{\sigma_{i}}$ to a more special position, that is, to to the boundary of the normal cone it lies in. It can now be seen that for every movement of a function value of a maximal chamber-say $\psi_{\sigma_{1}}$ - to a face of the normal cone associated with an edge, say $\psi_{\sigma_{12}}$, a movement of the other normal vector-here $\psi_{\sigma_{2}}$-contained in that cone to the same face is required in order to stay locally coherent.

The idea is now to produce a configuration of normal cones of the fibers such that for each cone corresponding to the starting values of the function $\psi$ no face is reachable by both the function values of the maximal chambers in a way such that the intermediate functions stay locally coherent.

Consider the "basket ball" with three segments in Fig. 6(a): the normal fan of a triangle in $\mathbb{R}^{3}$ intersected with the 2 -sphere. We take three perturbed copies of this configuration such that the superposition locally looks as in Fig. 6(b). The rounded triangle bounding the configuration sketches the normal cone of $\psi_{\sigma_{1}}$ over $\sigma_{123}$ (its exact shape is not important, it could have, for example, more than six extremal rays). Together with the three basket balls it provides the three triangular "prisons" which the function values are placed into. These function values are pairwise locally coherent because $\psi_{\sigma_{1}}$ and $\psi_{\sigma_{2}}$

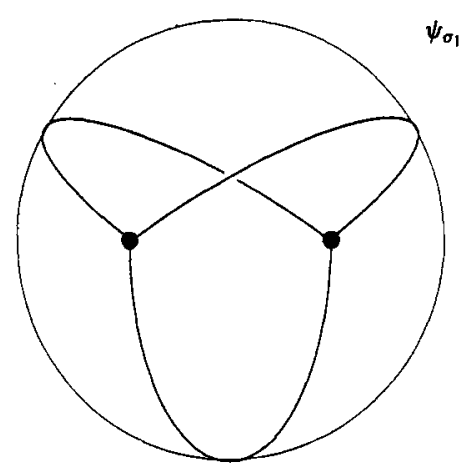

(a)

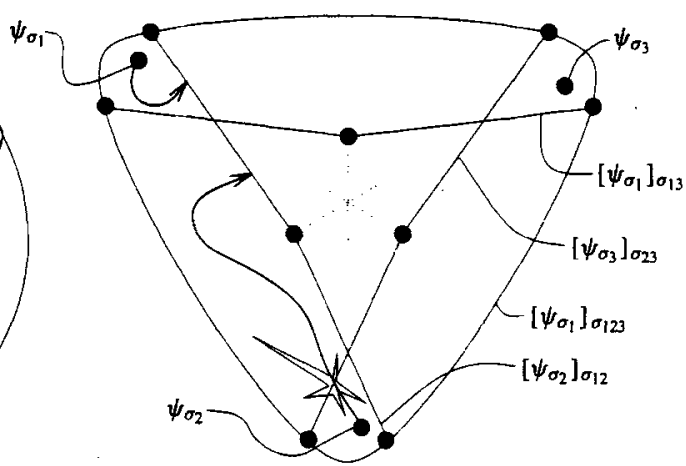

(b)

Fig. 6. The "basket-ball obstruction." 


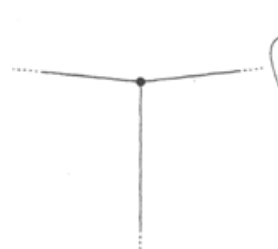

(a)

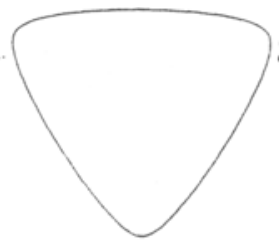

(b)

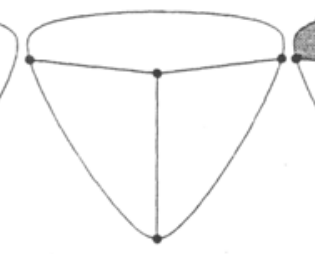

(c)

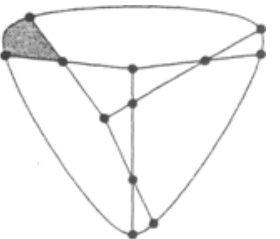

(d)

Fig. 7. A part of the normal fan over the vertex $\tau_{12}$ of $Q$ (a), over $\sigma_{123}$ (b), over the corresponding edge $\sigma_{12}$ (c), and over the adjacent two-dimensional chamber $\sigma_{1}$ (d). If $\psi_{\sigma_{1}}$ is chosen in the interior of the shaded cone, and if $\psi_{\sigma_{2}}$ and $\psi_{\sigma_{3}}$ are chosen analogously with respect to the rotational symmetry we get the basket-ball obstruction of Fig. 6.

lie in the same cone $\left[\psi_{\sigma_{1}}\right]_{\sigma_{12}}$ over $\sigma_{12}$, and so on. There is no possibility of pushing the function values to a more special position without violating the pairwise cone condition.

Assume, without loss of generality, $\psi_{\sigma_{1}}$ moves to a face of $\left[\psi_{\sigma_{1}}\right]_{\sigma_{12}}$ while no other function value has reached a more special position earlier. Then $\psi_{\sigma_{2}}$ has to move to the same face at the same moment-but then it must have passed over a face of $\left[\psi_{\sigma_{3}}\right]_{\sigma_{23}}$ in the meantime: a contradiction (see the "funny star-like thing," a flash where the contradiction occurs, in Fig. 6(b)).

In the same manner all possibilities of moving function values over the 2-chambers fail. Hence this provides an obstruction for homotopies on the starting function $\psi$ which we call the "basket-ball obstruction."

The configuration of Fig. 6 is realized by the following innocent-looking construction that is illustrated in Fig. 7.

- Let $\pi: \mathbb{R}^{5} \rightarrow \mathbb{R}^{2}$ be the projection to the last two coordinates.

- Put three triangles into $\mathbb{R}^{5}$ in the following way: each triangle projects down to one vertex of the triangle $Q$, such that the superposition of their normal fans in $\mathbb{R}^{3}$ (basket balls!) locally looks like the configuration inside $\left[\psi_{1}\right]_{\sigma_{123}}$ in Fig. $6(\mathrm{~b})$ - the local basket-ball obstruction.

- Let $\widetilde{P^{\text {deg }}}$ be their convex hull in $\mathbb{R}^{5}$. At this point the normal fan over $\sigma_{123}$ is the common refinement of the three basket balls (Corollary 2.5).

- Position a single vertex into $\mathbb{R}^{5}$ such that it projects to the 0 -cell $\sigma_{123}$ in the center of $\Gamma$. The resulting fiber over $\sigma_{123}$ will just be the convex hull of the old fiber over $\sigma_{123}$ and the new vertex. Choose the new vertex $v$ in such a way that its normal cone in the fiber realizes the cone $\left[\psi_{1}\right]_{\sigma_{123}}$ of Fig. $6(\mathrm{~b})$. (From the primal point of view we put the vertex "beyond" those faces of the fiber that have normal cones in the local basket-ball obstruction. Hence, in the new normal fan over $\sigma_{123}$ the local basket-ball obstruction is replaced by the normal cone of the new vertex.) The resulting polytope $P^{\mathrm{deg}}=\operatorname{conv}\left(\widetilde{P^{\text {deg }}}, v\right)$ has 10 vertices.

- Because of Proposition 2.2 the normal fans over the edges $\sigma_{i j}$ of $\Gamma$ are the common refinement of the normal fan over $\sigma_{123}$ and the normal fan over the corresponding vertex of $Q$. Over a vertex of $Q$ there is one basket ball and over $\sigma_{123}$ there is a fan that contains a cone that "locks" the basket-ball obstruction into one cone.

- Define the function values on $\sigma_{i}$ as in Fig. $6(\mathrm{~b})$ and the function values on $\sigma_{i j}$ somewhere inside the corresponding cones $\left[\psi_{\sigma_{i}}\right]_{\sigma_{i j}}=\left[\psi_{\sigma_{j}}\right]_{\sigma_{i j}}$. 
- Complete this function on the boundary of $Q$ (Proposition 2.8). This yields a tight locally coherent string that is not dominated by a coarser one, i.e., an isolated element in $\omega(P, \pi)$ (see Lemma 1.2(b)).

In Section 5 we present a version of $P^{\text {deg }}$ with explicit coordinates in $\mathbb{R}^{5}$. Moreover, we slightly perturb the vertices of $P^{\text {deg }}$ to get a simplicial, nondegenerate counterexample $P$. For each of them we provide another, simple way to see that it violates the Generalized Baues Conjecture.

\section{An Explicit Counterexample}

Throughout this section we use homogeneous coordinates in order to get a nice threefold rotational symmetry for $Q^{\text {deg }}$ and $Q$ without square roots. We use projections that delete the first three coordinates. The following list contains as rows the (homogeneous) coordinates for ten points in $\mathbb{R}^{5}$ in convex position:

\begin{tabular}{|c|c|c|c|c|c|c|}
\hline \multicolumn{7}{|c|}{$\begin{array}{l}\text { DIM }=6 \\
\text { CONE } \cdot \text { SECTION }\end{array}$} \\
\hline (1) & 1 & 0 & 0 & 1 & 0 & 0 \\
\hline$(2)$ & 0 & $3 / 2$ & 1 & 1 & 0 & 0 \\
\hline (3) & 0 & 1 & $3 / 2$ & 1 & 0 & 0 \\
\hline$(4)$ & 0 & 1 & 0 & 0 & 1 & 0 \\
\hline$(5)$ & 1 & 0 & $3 / 2$ & 0 & 1 & 0 \\
\hline$\left(\begin{array}{l}(6) \\
-1\end{array}\right.$ & $3 / 2$ & 0 & 1 & 0 & 1 & 0 \\
\hline$(7)$ & 0 & 0 & 1 & 0 & 0 & 1 \\
\hline$(8)$ & $3 / 2$ & 1 & 0 & 0 & 0 & 1 \\
\hline ( 9$)$ & 1 & $3 / 2$ & 0 & 0 & 0 & 1 \\
\hline$(10)$ & 2 & 2 & 2 & $1 / 3$ & $1 / 3$ & $1 / 3$ \\
\hline
\end{tabular}

The first nine rows correspond to the three triangles of the abstract construction in Section 4, the tenth one represents the additional vertex. The chamber complex of the projection to the last three coordinates is as in Fig. 5(a). The normal fans of the three triangles in $\mathbb{R}^{3}$ form the basket ball obstruction. The additional vertex yields the midpoint of the chamber complex and bounds the obstruction over the edges of the chamber complex. Figure 8 is an attempt to visualize the construction.

The above listing is in correct input format for the PORTA program by Christof [8]. This program easily produces a complete list of all 36 facets of $P$, and the vertex-facet incidence matrix in Fig. 10.

The following tight locally coherent string-where the faces $F_{1}^{\mathrm{deg}}, F_{2}^{\mathrm{deg}}, F_{3}^{\mathrm{deg}}$ (see Fig. 9) are given by their vertices labeled as in the listing above-correspond to the three given function values in Fig. 6 :

$$
\mathcal{F}_{0}^{\text {deg }}:=\{(1,4,10),(4,7,10),(7,1,10)\} .
$$




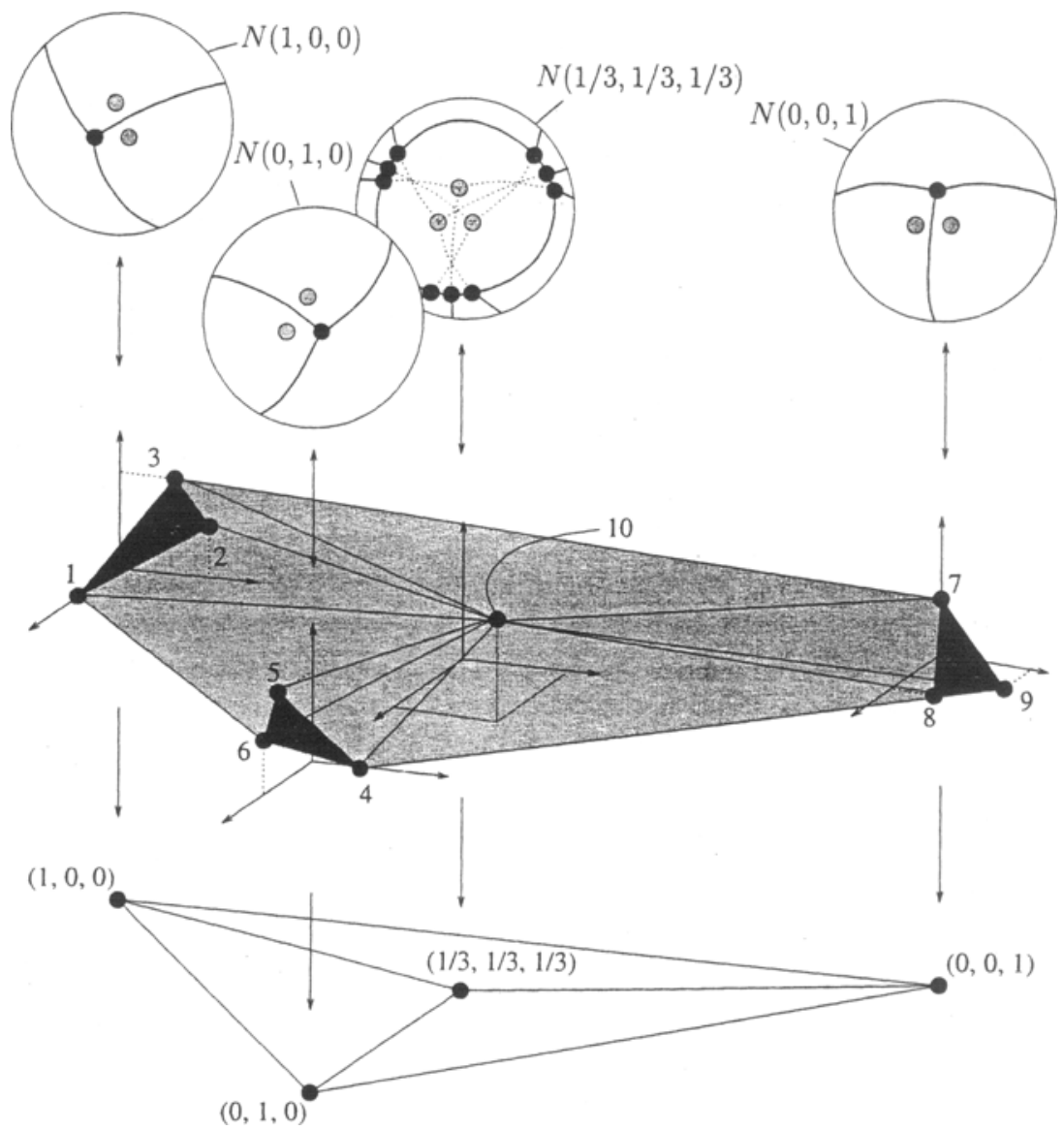

Fig. 8. A sketch of $\pi^{\text {deg }:} P^{\text {deg }} \rightarrow Q^{\text {deg }}$ : Over each vertex of $Q^{\text {deg }}$ one perturbed basket ball is positioned. Adding the tenth vertex in the middle provides a bounding cone around the basket-ball obstruction. (The gray vertices and the dotted lines are drawn to indicate the positions of the fans with respect to each other.) The 5-polytope $P^{\text {deg }}$ is the convex hull of the three dark triangles-each of them in an $\mathbb{R}^{3}$ over one vertex of $Q^{\text {deg }}$-and the additional vertex $(10)$ in the middle.

where the actual string contains all the nonempty faces of the three triangles that are listed.

Once we have this, it is very easy to see independently from Section 4 that this is a counterexample to the Generalized Baues Conjecture. To form a strictly coarser string we must replace at least one of the faces $F_{1}^{\mathrm{deg}}, F_{2}^{\mathrm{deg}}, F_{3}^{\mathrm{deg}}$ in $\mathcal{F}_{0}^{\mathrm{deg}}$ by a face $\hat{F}_{i}^{\mathrm{deg}}$ of $P^{\mathrm{deg}}$ that contains $F_{i}^{\text {deg }}$. This can be described by adding one or more vertices to $F_{i}^{\text {deg }}$ such that we get a face. From the definition of a locally coherent string it follows that a new vertex $v$ has to be added (combinatorially) to all faces of $\mathcal{F}_{0}^{\text {deg }}$ whose projection contains $\pi(v)$.

From the vertex-facet incidence matrix we can compute for each face $F$ in $\mathcal{F}_{0}^{\text {deg }}$ all sets $V$ of vertices in $P^{\text {deg }} \backslash F$ such that vert $(F) \cup V$ are the vertices of a face in $P^{\text {deg }}$. They 


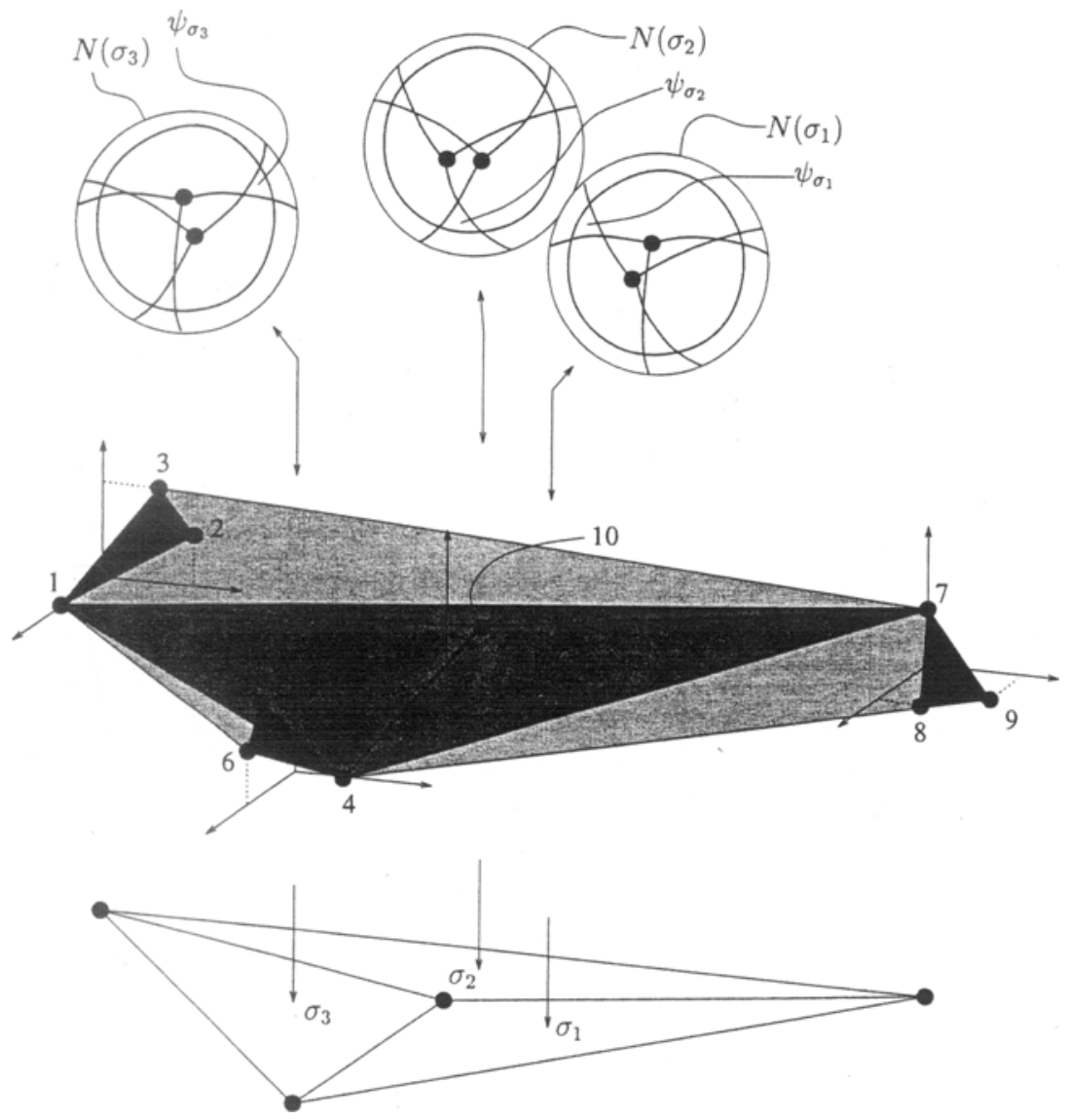

Fig. 9. The medium-dark triangles correspond to the isolated locally coherent string $\{(1,4,10),(4,7,10)$, $(7,1,10)\}$ that is defined by the function values $\psi_{\sigma_{1}}, \psi_{\sigma_{2}}$, and $\psi_{\sigma_{3}}$ for the chambers $\sigma_{1}, \sigma_{2}$, and $\sigma_{3}$.

correspond exactly to the faces in the link of $F$ in $P^{\mathrm{deg}}$ denoted by $l k(F):=l k_{P \operatorname{deg}}(F)$. It turns out that all links are 4-gons, namely

$$
\begin{aligned}
& l k(1,4,10)=(2-9-8-6-2), \\
& l k(4,7,10)=(5-3-2-9-5), \\
& l k(7,1,10)=(8-6-5-3-8) .
\end{aligned}
$$

Because of the rotational symmetry it suffices to test the vertices in $l k(1,4,10)$. For example adding vertex 2 to the face $(1,4,10)$ requires adding vertex 2 to the face $(7,1,10)$ because $\pi(7,1,10)$ contains $\pi(2)$-but vertex 2 is not contained in the link of $(7,1,10)$. Analogous contradictions occur in all other cases. This proves that $\mathcal{F}_{0}^{\text {deg }}$ is in fact an isolated element in $\omega\left(P^{\text {deg }}, \pi\right)$. This example corresponds exactly to the construction at the end of Section 4. 


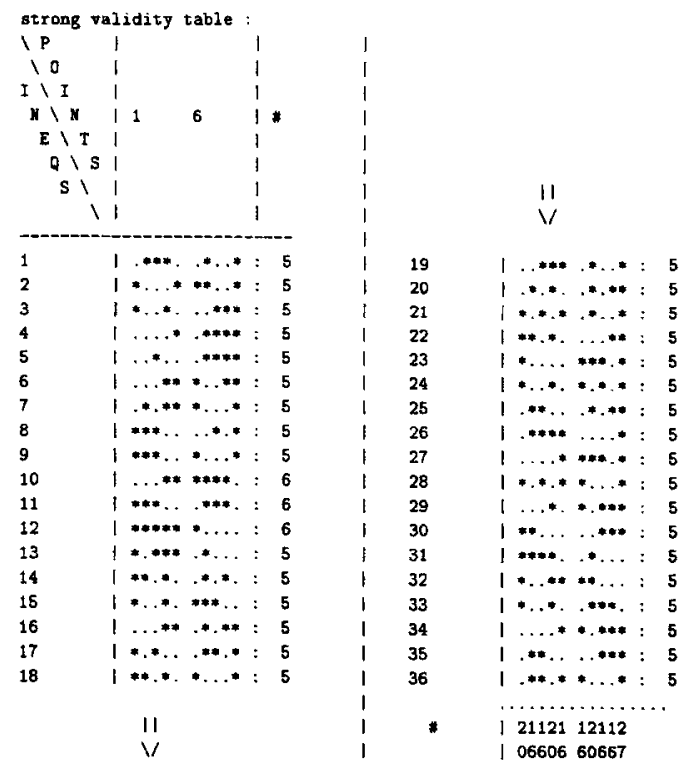

Fig. 10. The vertex-facet incidence matrix of $P^{\text {deg }}$.

The coordinates of $P^{\text {deg }}$ can be slightly perturbed in order to make the projection nondegenerate. We claim that the following listing contains the coordinates of a simplicial, nondegenerate counterexample $P$ :

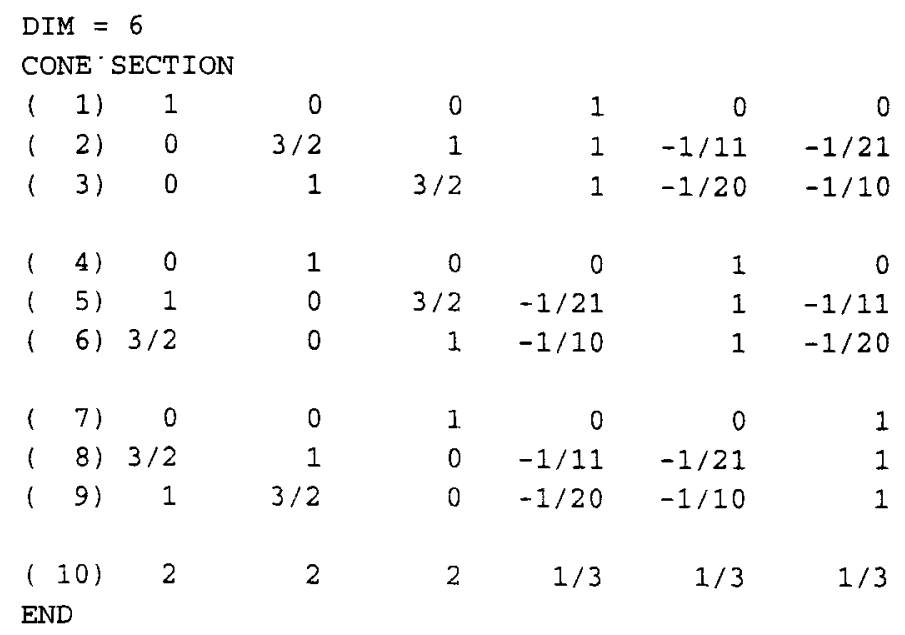

All the vertices of $P$ project to pairwise different points in the plane. We again inspect the vertex-facet incidence matrix, see Fig. 12. Each facet has exactly five vertices, so $P$ is a simplicial polytope. Consider the chamber complex of the projection in Fig. 11-a computer-generated drawing which also shows that $P$ is 2 -neighborly. The projections of the three faces $(1,4,10),(4,7,10)$, and $(7,1,10)$ do not cover $Q$. However, for 


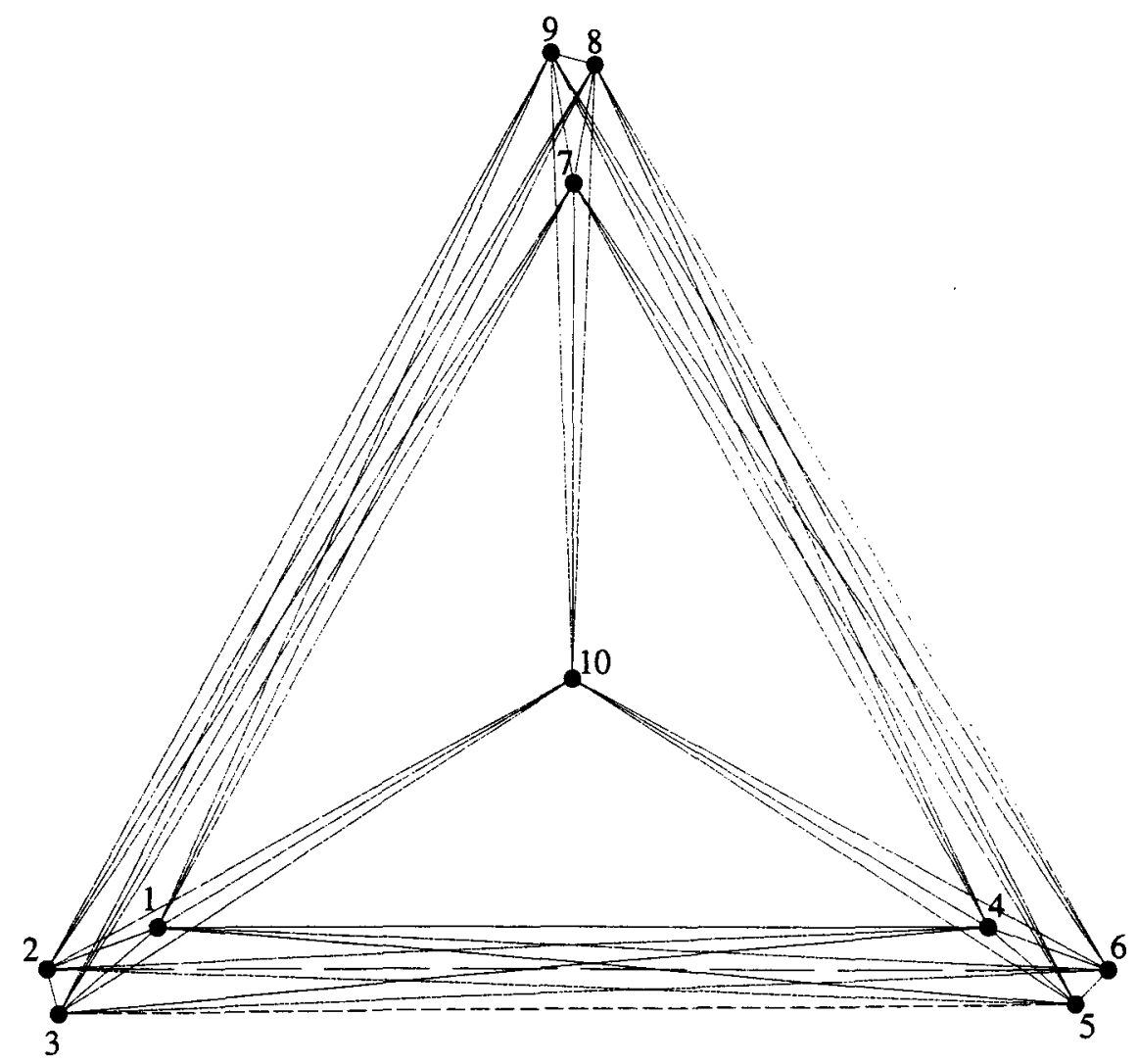

Fig. 11. The chamber complex of $\pi$.

chambers that are not covered we find, for example, the following tight completion:

$$
\begin{aligned}
\mathcal{F}_{0}:= & \{(1,4,10),(1,2,4),(2,3,4),(3,4,5), \\
& (4,7,10),(4,5,7),(5,6,7),(6,7,8), \\
& (7,1,10),(7,8,9),(8,9,1),(9,1,2)\} .
\end{aligned}
$$

This is not an isolated element in $\omega(P, \pi)$, because there are local changes possible on the new faces. For example, the faces $(1,2,4)$ and $(2,3,4)$ are dominated by $(1,2,3,4)$, etc. However, a local change of $(1,4,10),(4,7,10)$, or $(7,1,10)$ is not possible. To see this, we first check that no facet of $P$ contains more than one of these three faces. Consider again Fig. 11. If, without loss of generality, we take any face $F$ in $P$ that contains $(1,4,10)$ we observe that some new edge of $F$ projects into the interior of $\pi(4,7,10)$ or $\pi(7,1,10)$. (The link of $(1,4,10)$ is again $(2-9-8-6-2)$, etc.) For example, if we replace $(1,4,10)$ by $(1,2,4,10)$, then the projection of the new edge $(2,10)$ cuts through the interior of $\pi(7,1,10)$. Thus we have produced overlapping projections, a 


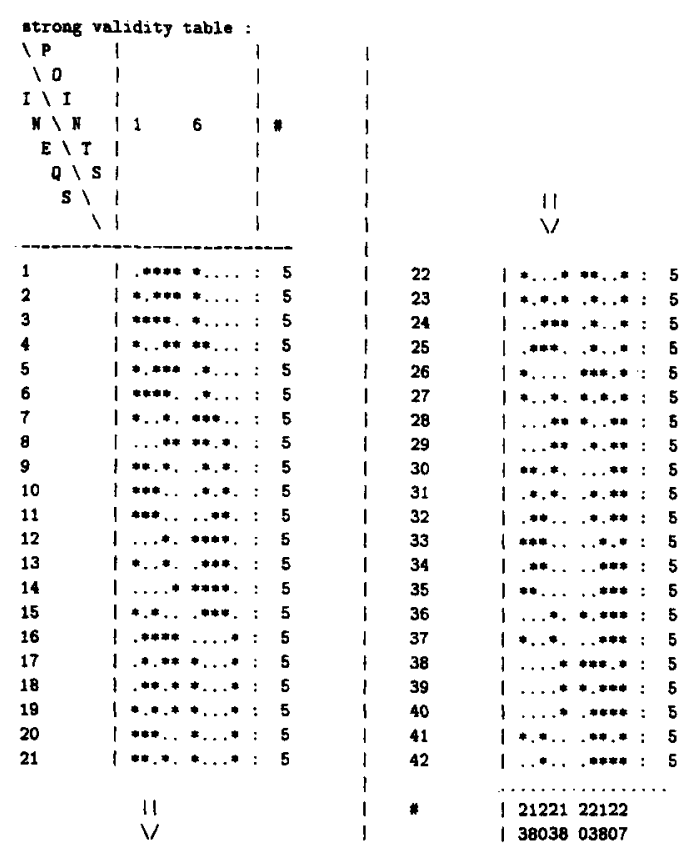

Fig. 12. The vertex-facet incidence matrix of $P$.

contradiction to the fact that every locally coherent string defines a polyhedral subdivision after projection.

We see that any locally coherent string in the connected component of $\mathcal{F}_{0}$ must contain the three faces $(1,4,10),(4,7,10)$, and $(7,1,10)$. However, obviously there is the following locally coherent string where the face $(1,4,7)$ replaces the three "rigid" faces:

$$
\begin{aligned}
\mathcal{F}_{1}:= & \{(1,4,7),(1,2,4),(2,3,4),(3,4,5), \\
& (4,5,7),(5,6,7),(6,7,8), \\
& (7,8,9),(8,9,1),(9,1,2)\} .
\end{aligned}
$$

Thus we conclude that there are at least two connected components in $\omega(P, \pi)$, in contradiction to the Generalized Baues Conjecture.

\section{Acknowledgments}

We thank Jürgen Richter-Gebert and Nikolai Mnëv for helpful discussions.

\section{References}

1. J. F. Adams. On the cobar construction. Proceedings of the National Academy of Science, 42:409-412, 1956. 
2. H. J. Baues. Geometry of loop spaces and the cobar construction. Memoirs of the American Mathematical Society, 25(230):171, 1980.

3. L. J. Billera, 1. M. Gel'fand, and B. Sturmfels. Duality and minors of secondary polyhedra. Journal of Combinatorial Theory, Series B, 57:258-268, 1993.

4. L. J. Billera, M. M. Kapranov, and B. Sturmfels. Cellular strings on polytopes. Proceedings of the American Mathematical Society, 122:549-555, 1994.

5. L. J. Billera and B. Sturmfels. Fiber polytopes. Annals of Mathematics, 135:527-549, 1992.

6. A. Björner. Essential chains and homotopy types of posets. Proceedings of the American Mathematical Society, 402:1179-1181, 1992.

7. A. Björner. Topological methods. In R. Graham, M. Grötschel, and L. Lovász, editors, Handbook of Combinatorics, pp. 1819-1872. North-Holland, Amsterdam, 1995.

8. T. Christof. Porta-a polyhedron representation transformation algorithm v. 1.2.1. Available from the ZIB electronic library ELIB via el ibe z ib-berlin. de or by anonymous ftp from elib. $z i b$-berlin. de, directory / pub/mathprog/polyth.

9. P. H. Edelman and V. Reiner. Visibility complexes and the Baues Problem for triangulations in the plane. Preprint 1995.

10. H. Edelsbrunner and N. R. Shah. Incremental topological flipping works for regular triangulations. Proceedings of the 8th Annual ACM Symposium on Computational Geometry, Berlin, pp. 43-52. ACM Press, New York, 1992.

11. B. Joe. Three-dimensional triangulations from local transformations. SIAM Journal of Scientific Statistical Computation, 10:718-741, 1989.

12. B. Joe. Construction of three-dimensional Delaunay triangulations using local transformations. Computer Aided Geometric Design, 8:123-142, 1991.

13. R. D. MacPherson. Combinatorial differential manifolds. In L. R. Goldberg and A. V. Phillips, editors, Topological Methods in Modern Mathematics: a Symposium in Honor of John Milnor's Sixtieth Birthday, Stony Brook, NY, 1991, pp. 203-221. Publish or Perish, Houston, TX, 1993.

14. N. E. Mnëv and G. M. Ziegler. Combinatorial models for the finite-dimensional Grassmannians. Discrete \& Computational Geometry, 10:241-250, 1993.

15. A. Nabutovsky. Extremal triangulations of manifolds. Preprint, 1994.

16. B. Sturmfels. Fiber polytopes: a brief overview. In M. Yoshida, editor, Special Differential Equations, pp. 117-124. Kyushu University, Fukuoka, 1991.

17. B. Sturmfels and G. M. Ziegler. Extension spaces of oriented matroids. Discrete \& Computational Geometry, 10:23-45, 1993.

18. G. M.Ziegler. Lectures on Polytopes. Graduate Texts in Mathematics, vol. 152. Springer-Verlag, New York, 1995. Updates, corrections, and more available at ht tp://www . math. tu-berlin. de/ ziegler.

Received February 15, 1995, and in revised form August 14, 1995. 